DOI: 10.29295/2311-7257-2019-96-2-362-368

УДК 539.3

\title{
Сизова Н.Д.
}

Харьковский национальный университет строительства и архитектуры (ул. Сумская, 40, Харьков, 61002, Украина; e-mail: contact@kn-it.info; orcid.org/0000-0001-9406-4926)

\section{ЧИСЛЕННО-АНАЛИТИЧЕСКИЕ РЕШЕНИЯ ТЕМПЕРАТУРНЫХ ЗАДАЧ В ОБЛАСТЯХ СЛОЖНОЙ ФОРМЫ}

Приводятся исследования температурных задач в областях сложной геометрической формы, которые основаны на совместном применении дифференциально - разностного подхода и метода Rфункций (RFM). Решения температурных задач представлены в аналитическом виде с возможностью определения численных решений с привлечением современных методов.

Ключевые слова: температурные задачи, моделирование, метод R-функций, разностные схемы, алгоритм, вычислительный эксперимент.

Введение. В современном градостоительстве получают широкое распространений новые геометрические образы зданий и внутренних конструкций, которые представляются сложными неклассическими объектами, подлежащими всестороннему исследованию [1-6]. Одним из таких направлений является моделирование и исследование температурных полей. Эта задача - один из наиболее важных факторов, влияющих на надежность и устойчивость работы конкретных элементов, так как изменение температуры вызывает и изменение свойств различных материалов, входящих в состав общей конструкции.

Экспериментальные исследования температурных полей во многих случаях для конструктивных элементов неприемлемы из-за сложности их геометрических характеристик, внешних и внутренних температурных воздействий, или же физические эксперименты являются достаточно дорогостоящими. В силу этого актуальным является определение температурных полей конструктивных элементов аналитическими и численными методами.

Решение теплофизической задачи позволяет получить достоверную и объективную информацию о распределении температуры внутри конструктивного элемента, заменить экспериментальную разработку режимов работы элементов расчетным проектированием, прогнозировать тепловые процессы, рассчитывать оптимальные режимы нагрева конструкции.

В задачах строительства предъявляются повышенные требования к точности определения температурных полей исследуемых элементов и узлов, поэтому объяснимо стремление создать универсальные подходы и высокоточные алгоритмы решения таких задач.

Универсальные подходы предполагают получения решения в аналитическом, графическом или численном виде [7- 17]. Промежуточными этапами определения решений являются численно-аналитические методы $[6,10,11]$.

Аналитические решения в виде формульных соотношений исследования той или иной задачи (а это технические, экономические, социальные и др.), позволяют, как правило, приводить решения для ограниченного класса задач. Если речь идет о получении результата в виде числа, то применяется численный анализ моделей - инструментарий исследования современных прикладных задач [12 -14].

Численно-аналитический подход, основанный на теории $\mathrm{R}$ - функций [15- 17], позволяет представлять решение в аналитическом виде для произвольных геометрических объектов, находящихся под воздействиями различной физической природы, и привлекать современные численные методы.

Целью работы является развитие конструктивных средств теории R-функций для пространственных краевых задач 
теплопроводности. В связи с этим задачей исследования является исследование математических моделей теплопроводности, описываемых системами линейных дифференциальных уравнений, разработка методики решения пространственных стационарных задач теплопроводности, основанная на совместном применении дифференциально-разностного метода и теории R-функций.

Анализ литературных источников. Основное дифференциальное уравнение в частных производных, описывающих стационарное распространение тепла в трехмерном анизотропном теле $\Omega$, ограниченном кусочно-гладкой поверхностью $\mathrm{S}$, с постоянными теплофизическими характеристиками, имеет вид [7-9]

$$
\begin{aligned}
& M u=-\sum_{l=1}^{3} a_{l l} \frac{\partial}{\partial x_{l}}\left(\frac{\partial u}{\partial x_{1}}\right)+a_{0} u=f(x), \\
& x=\left(x_{1}, x_{2}, x_{3}\right) \in \Omega, \\
& u(x) \in H^{1}(\Omega, M)=\left\{u \in H^{1}(\Omega), M u(x) \in\right. \\
& \left.L^{2}(\Omega)\right\}, a_{0}>0, f(x) \in L^{2}(\Omega) .
\end{aligned}
$$

Помимо уравнения (1) на поверхности $\mathrm{S}$ задаются граничные условия

$$
\begin{gathered}
\left.u(x)\right|_{S}=\psi(x), \\
N u=\sum_{k, l}^{3} b_{k l} \frac{\partial u}{\partial x_{l}} \cos \left(v_{1}, x_{k}\right)-b_{0} u=0 \\
x \in\left(x_{1}, x_{2}\right) \in S, \quad \mathrm{~S}=\stackrel{\substack{k=1 \\
r}}{ } S_{k} \\
b_{k l}(x), b_{0}(x) \in H^{1 / 2}(S) .
\end{gathered}
$$

Соотношение (3) в общем случае не является однородным, но однородные граничные условия (3) можно получить с помощью соответствующей замены.

Заметим, что (3) является общим граничным условием, которое за счет выбора коэффициентов $b_{k l}, b_{0}$ приводит к граничным условиям первого, второго и третьего типа; комбинируя на различных поверхностях $S_{i}$ граничные условия разных типов, приходим к смешанным граничным задачам.

Точные аналитические решения данного класса задач построены для классических областей, и поэтому большое значение имеет привлечение для исследования решений в областях произвольной формы приближенных численных методов [7-14].
Для исследования задач (1-3) наиболее развиты подходы, основанные на методах конечных разностей (МКР) и метода конечных элементов (МКЭ) [12-14] и др.

Предметом исследования является применение теория R-функций к изучению математических моделей температурных задач. Приведем соответствующие решения, названные структурными (GSS) [15-17].

Структурные модели, построенные в функциональных пространствах, являются общими и служат основой для составления моделей исследования определенного класса задач и используются для качественного анализа решений этого класса задач, т. е. для определения общих свойств решений [15]. Эти модели позволяют учесть необходимые с физической точки зрения данные, содержащиеся в математической постановке исходной задачи, провести анализ и оптимизацию физико-механических систем по заранее заданному критерию оптимальности.

Приведем основные моменты теории R-функций (RFM) [15], которая привнесла методы математической физики конструктивные элементы алгебры логики. Это в свою очередь позволило сформулировать понятие структуры решения (GSS) (т.е. формы, в которой отыскивается решение) как функциональное соотношение

$$
U=B(\Phi),
$$

где $U$ - решение задачи, $B$ - известный оператор, учитывающий граничные условия краевой задачи, определяемый на множестве $M$, а элемент $\Phi$ выбирается так, чтобы наилучшим образом (в том или ином смысле) удовлетворить исходному уравнению.

Основная идея построения структурных формул, учитывающих граничные условия, состоит в разложении в ряд искомого решения по степеням функции $\omega$ или $\omega_{1}$, где $\omega, \omega_{1}$ - левые части нормализованных уравнений границы области $\mathrm{S}(\partial \Omega)$ или их участков.

Пусть граница $\partial \Omega$ области $\Omega$ будет $C^{\infty}$ - многообразием и описывается $\omega(x) \in C^{\infty}\left(R^{n}\right), \quad x \in R^{n}$, 
при этом

$$
x=\left\{x_{1}, x_{2}, \ldots, x_{n}\right\},
$$

$$
\begin{array}{rll}
\omega(x)>0 & \text { для } & x \in \Omega, \\
\omega(x)=0 & \text { для } & x \in \partial \Omega, \\
\omega(x)<0 & \text { для } & x \notin \Omega, \\
\left.\frac{\partial \omega(x)}{\partial v}\right|_{\partial \Omega}=1, & &
\end{array}
$$

где $v$ - внутренняя нормаль. Построение такой функции $\omega(x)$ осуществляется по методике [15] с использованием $\mathrm{R}$ - операций, это один из этапов нахождения решения исходной задачи.

Приведем структурные формулы для основных краевых задач математической физики (для уравнений 2-го порядка) $[3,9]$.

Для задачи Дирихле, рассматриваемой в некоторой области $\Omega$ с границей $\Gamma$,

$$
\begin{aligned}
& A u=f(x), \\
& \left.u\right|_{\Gamma}=\phi(x),
\end{aligned}
$$

где $\mathrm{A}$ - дифференциальный оператор эллиптического типа, $f(x), \varphi(x)$ - известные функции, определяющие соответственно правую часть уравнения и граничные условия имеет вид, следующее соотношение при любом выборе $\Phi \in \mathrm{C}^{2}(\Omega)$ приводит к выражению

$$
\mathrm{u}=\omega \Phi+\varphi .
$$

Представляя неопределенную компоненту $\Phi(x)$ разложением по функциям некоторой базисной системы $\left\{\chi_{i}(x)\right\}_{i=1}^{n}$, получаем аппроксимацию решения краевой задачи Дирихле в виде

$$
\begin{aligned}
& \Phi(x)=\sum_{i=1}^{n} C_{i} \chi_{i}(x), \\
& u(x)=\sum_{i=1}^{n} C_{i} \phi_{i}(x)+\phi_{0}(x), \\
& \phi_{i}(x)=\omega(x) \chi_{i}(x),
\end{aligned}
$$

где $\mathrm{C}_{\mathrm{i}}$ - неизвестные константы, которые определяются из условия минимума функционала вариационной задачи, эквивалентной исходной краевой задаче $[18,19]$.

Для задачи Неймана граничные условия имеют вид

$$
\left.\frac{\partial u}{\partial v}\right|_{\Gamma}=g(x)
$$

где $\mathrm{g}(\mathrm{x})$ - заданная функция.

Структурную формулу для задачи ( Неймана можно представить выражением

$$
u=P_{0}(x)-\omega D_{1} P_{0}(x)+\omega^{2} P_{1}(x)+\omega g(x) .
$$

Нетрудно заметить, что приведенные структуры решения краевых задач Дирихле и Неймана точно удовлетворяют граничным условиям независимо от выбора функций $\mathrm{P}_{0}(x)$ и $\mathrm{P}_{1}(x)$.

Приведем структурную формулу решения смешанной задачи математической физики, граничные условия для которой имеют вид

$$
\left.u\right|_{\Gamma_{1}}=\phi(x),\left.\left(\frac{\partial u}{\partial v}+h_{0} u\right)\right|_{\Gamma_{2}}=g(x),
$$

где $\varphi(x)$ и $g(x)$ - заданные на границе функции, $\mathrm{h}_{0}-$ известная функция, $\Gamma_{1}$ и $\Gamma_{2}$ - участки границы области $\Omega$, уравнения для которых записываются функциями $\omega_{1}$ и $\omega_{2}$ соответственно:

$u=\omega_{1} P_{0}(x)+\frac{\omega_{1} \omega_{2}}{\omega_{1}+\omega_{2}}\left[g(x)+\omega_{2} P_{2}-\right.$
$D_{1}^{(2)}\left(\omega_{1} P_{0}(x)\right)-D_{1}^{(2)} \phi(x)-h_{0} \omega_{1}-$
$\left.h_{0} \phi(x)\right]+\phi(x)$,

при этом $D_{1}^{(2)}=\frac{\partial}{\partial x_{1}} \frac{\partial \omega_{2}}{\partial x_{1}}+\frac{\partial}{\partial x_{2}} \frac{\partial \omega_{2}}{\partial x_{2}}$.

В качестве функций $\vec{\Phi}$ и Р могут выбираться классические полиномы, полиномы с локальными носителями или специальные функции.

Поэтому предлагается подход к исследованию пространственных краевых задач теплопроводности в области $\Omega$ (ограниченной $\quad\left(\mathrm{a}_{1} \leq \mathrm{x}_{1} \leq \mathrm{a}_{2}, \quad \mathrm{~b}_{1} \leq \mathrm{x}_{2} \leq \mathrm{b}_{2}\right.$, $\left.\mathrm{c}_{1} \leq \mathrm{x}_{3} \leq \mathrm{c}_{2}\right)$, который использует применение по одной из переменных дифференциально-разностного подхода $[9,12]$ и теории R-функций, и, в частности, когда поперечное сечение области $\Omega$ не является постоянным вдоль одной из переменных.

Разобьем отрезок $\left[c_{1}, c_{2}\right]$ изменения переменной $x_{3}$ на $n+1$ произвольных частей плоскостями $x_{3}=x_{3 \mathrm{j}}(j=1,2, \ldots, n) \mathrm{c}$

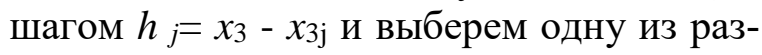
ностных аппроксимаций для производных по переменной $x_{3}$, например, в виде [9]

$$
\begin{gathered}
\frac{\partial^{2} u}{\partial x_{3}^{2}}=\frac{1}{\bar{h}_{j}}\left[\frac{u_{j+1}-u_{j}}{h_{j+1}}-\frac{u_{j}-u_{j-1}}{h_{j}}\right], \\
\bar{h}_{j}=\frac{h_{j}+h_{j+1}}{2} .
\end{gathered}
$$

В результате применения к уравнению (1) и граничным условиям (2) разностной аппроксимации (8) понижается 
размерность исходной краевой задачи и получается следующая система дифференциальных уравнений в частных производных

$$
\begin{aligned}
& M u_{j}=-\sum_{l=1}^{2} a_{l l} \frac{\partial}{\partial x_{1}}\left(\frac{\partial u_{j}}{\partial x_{1}}\right)+a_{0} u_{j}+ \\
& +B_{j} u_{j+1}-A_{j} u_{j}+C_{j} u_{j-1}=f_{j}(x) \text {, } \\
& x=\left(x_{1}, x_{2}\right) \in \Omega_{j}, \quad u_{j}\left(x_{1}, x_{2}\right)= \\
& u\left(x_{1}, x_{2}, x_{3 j}\right), j=1,2, \ldots, n \text {, } \\
& N u_{j}=\sum_{k, l}^{2} b_{k l} \frac{\partial u_{j}}{\partial x_{l}} \cos \left(v_{1}, x_{k}\right)- \\
& -b_{0} u_{j}=0 \\
& \text { где } B_{j}=\frac{a_{33}}{h_{j+1} \bar{h}_{j}}, A_{j}=-\frac{a_{33}}{\bar{h}_{j}}\left(\frac{1}{\overline{h_{j+1}}}+\frac{1}{\bar{h}}\right) \text {, } \\
& C_{j}=a_{33} / h_{j} \overline{h_{j}} \text {. }
\end{aligned}
$$$$
\text { Систему (9) - (10) можно привести к }
$$
системе дифференциальных уравнений для $\mathrm{n}$ несвязанных между собой двумерных задач [9], если введем $n$ - мерные векторы $U$ и $f(x)$, а также матрицу $n$ - го порядка $T$

$$
\begin{aligned}
& U=\left\|\begin{array}{l}
u_{1} \\
u_{2} \\
\cdots \\
u_{n}
\end{array}\right\|, \\
& f(x)=\left\|\begin{array}{l}
f_{1}(x)+C_{1} \psi_{1}\left(x_{1}, x_{2}\right) \\
f_{2}(x) \\
\ldots \ldots \ldots \ldots \ldots \ldots \ldots \ldots \ldots \ldots \ldots \ldots \ldots \ldots \ldots \ldots \ldots \\
f_{n}(x)+B_{n} \psi_{2}\left(x_{1}, x_{2}\right)
\end{array}\right\|,
\end{aligned}
$$

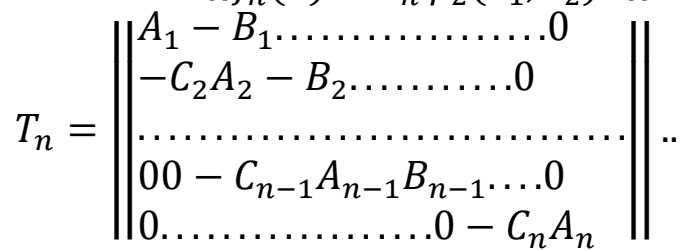

При этом матрица $T_{n}$ является якобиевой матрицей простой структуры, не имеет кратных корней и ее можно представить в виде

$$
T_{n}=R_{n}^{-1} \Lambda_{n} R_{n},
$$

где $\Lambda_{n}=\left\{\lambda_{1}, \lambda_{2}, \ldots \lambda_{n}\right\}$ - диагональная матрица $n$ - го порядка, элементами которой являются собственные значения матрицы $T_{n}$.

Задача на определение собственных значений матрицы $T_{n}$ эквивалентна следующей разностной краевой задаче типа Штурма-Лиувилля

$$
\begin{gathered}
r_{j+1}-r_{j}\left(1-\frac{h_{j+1}}{h_{j}}\right)+r_{j-1} \frac{h_{j+1}}{h_{j}}+\lambda h_{j} h_{j+1} r_{j} \\
=0,
\end{gathered}
$$

$r_{0}=r_{n+1}=0, \quad(j=1,2, \ldots n)$,

которая записывается системой разностных уравнений

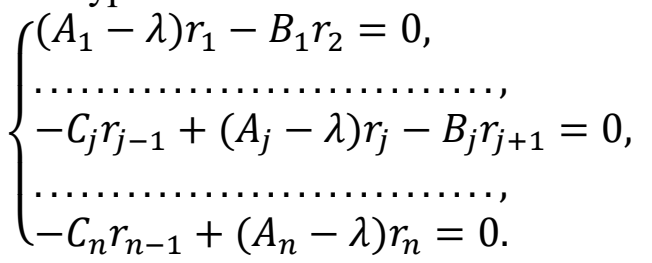

Характеристический многочлен в (13) раскрывается по следующим рекуррентным формулам

$D_{0}(\lambda)=1$,

$D_{1}(\lambda)=A_{1}-\lambda$,

$D_{j}(\lambda)=\left(A_{1}-\lambda\right) D_{j-1}(\lambda)-B_{j-1} C_{j} D_{j-2}(\lambda)$,

$(j=1,2, \ldots n)$.

Корни алгебраического уравнения (14) $D_{n}(\lambda)$ и являются собственными числами матрицы $T_{n}$. Преобразующая матрица $R_{n}^{-1}$ может быть составлена из линейно- независимых собственных векторов $r_{j s}$, соответствующих различным собственным значениям $\lambda_{\text {j. }}$.

Явная формула собственных векторов имеет вид

$$
\begin{aligned}
& r_{j s}=B_{1}^{-1} B_{2}^{-1} \ldots B_{j-1}^{-1} D_{j-1}\left(\lambda_{s}\right), \\
& (j, s=1,2, \ldots, n) .
\end{aligned}
$$

Пусть $U=R_{n} u, F\left(x_{1}, x_{2}\right)=R_{n} f$, тогда, учитывая соотношения (11) - (14), приходим к $n$ не связанным между собой двумерным краевым задачам

$$
\begin{gathered}
M U_{j}=-\sum_{i=1}^{2} a_{l l} \frac{\partial}{\partial x_{l}}\left(\frac{\partial U}{\partial x_{l}}\right)+\left(a_{0}+\lambda_{j}\right) U_{j}=F_{j}, \\
\left.N U_{j}\right|_{S}=0, \\
U_{j} \in H^{1}\left(\Omega_{j}, M\right)=\left\{\begin{array}{c}
U_{j} \mid U_{j} \in H^{1}\left(\Omega_{j}\right), \\
M U \in L^{2}\left(\Omega_{j}\right)
\end{array}\right\} .
\end{gathered}
$$

Перейдем к вопросу решения задачи (15) - (16) для каждого значения на каждом шаге $j$.

Рассмотрим случай, когда для задачи (15) - (16) выполнены условия применимости вариационного принципа [19]. Тогда задача (15) - (16) эквивалентна следующей вариационной задаче

$U_{j}^{*} \in W\left(\Omega_{j}\right)$,

$W\left(\Omega_{j}\right)=\left\{U_{j}\left|U_{j} \in H^{1}\left(\Omega_{j}, M\right), N U_{j}\right|_{S}=0\right\}$, $I\left(U_{j}^{*}\right)=\min I\left(U_{j}\right)$,

где $\mathrm{H}^{1}\left(\Omega_{j}, M\right)$ - область определения задачи $(15)-(16), U_{j}^{*}$ - элемент, минимизирующий функционал $I\left(U_{j}\right)$. При довольно 
общих предположениях относительно краевой задачи (15) -(16) элемент $U_{j}$ оказывается близким к $U_{j}^{*}$ в метрике $\mathrm{H}^{1}\left(\Omega_{j}, M\right)$.

Для нахождения элемента $U_{j n}$, который аппроксимирует элемент $U_{j}^{*}$ минимизирующего функционал $I\left(U_{j}\right)$, заменим бесконечномерную вариационную задачу (17) приближенной конечномерной задачей.

Эффективными методами такой замены являются методы Ритца, БубноваГалеркина и др.

Аппроксимирующий элемент $U_{j n}$ представляется формулой

$$
U_{j n}\left(x_{1}, x_{2}\right)=\sum_{i=1}^{n} C_{i} \phi_{i}\left(x_{1}, x_{2}\right)
$$

где $\left\{\phi_{i}\left(x_{1}, x_{2}\right)\right\}_{1}^{n}$ - координатная последовательность, которая предполагается известной, она строится с помощью структурного метода, основой которого является метод R-функций [15], Ci - неизвестные, определяемые из условий минимума функционала (17).

Структурный метод дает функциональное представление координатных функций (18), удовлетворяющих граничным условиям (16), в виде

$\phi_{i}\left(x_{1}, x_{2}\right)=\mu_{0} \chi_{i}+\sum_{k=1}^{r} \mu_{k} G_{k}\left(\mu_{0} \chi_{k}\right)$, (19) где $\mu_{0}, \mu_{k}$ - функциональные коэффициенты, построенные с помощью нормализованных функций $\omega=\omega(x)$ в виде единого аналитического выражения уравнения участков $S_{k}$ граничного контура $S$.

Это дифференциальное представление дает возможность [15]:

- учитывать на аналитическом уровне геометрию области $\Omega$;

- учитывать на участках ее границы $S_{k}$ граничные условия (16);

- позволяет учесть (за счет произвола в выборе функций $\chi_{i}$ ) имеющуюся априорную информацию о точном решении задачи;

- позволяет приблизиться к точному решению $\mathrm{U}_{\mathrm{T}}$ в метрике соответствующего функционального пространства $\eta$

$$
\left\|U_{j n}-U_{T}\right\|<\varepsilon
$$

Таким образом, если получено решение задачи (15)-(16), то решение исходной задачи (1) - (3) на каждом $j$ шаге изменения переменной $x_{3}$ может быть представлено в виде

$$
\begin{gathered}
u_{j}\left(x_{1}, x_{2}\right)=\sum_{k=1}^{n} U_{k}\left(x_{1}, x_{2}\right) B_{1}^{-1} B_{2}^{-1} \ldots \\
\ldots B_{j-1}^{-1} D_{j-1}\left(\lambda_{k}\right) .
\end{gathered}
$$

Результаты исследования. Рассмотрим пространственную стационарную задачу теплопроводности для призматического тела $\Omega \quad\left(0 \leq x_{1} \leq a_{1}\right.$, $0 \leq x_{2} \leq a_{2}, \quad 0 \leq x_{3} \leq a_{3}$, ограниченного плоскостями $x_{3}=0, x=c_{3}$ (рис. 1) (все переменные безразмерны):

$$
\Delta T\left(x_{1}, x_{2}, x_{3}\right)=\frac{\partial^{2} T}{\partial x_{1}^{2}}+\frac{\partial^{2} T}{\partial x_{2}^{2}}+\frac{\partial^{2} T}{\partial x_{3}^{2}}=0
$$$$
x \in\left(x_{1}, x_{2}, x_{3}\right) \in \Omega \text {, }
$$

на границе $\mathrm{S}$ заданы следующие граничные условия

$$
\left.T\right|_{S}=f\left(x_{1}, x_{2}, x_{3}\right),
$$

где $T\left(x_{1}, x_{2}, x_{3}\right)$ - искомая температура, $f\left(x_{1}, x_{2}, x_{3}\right)$ - заданная функция,

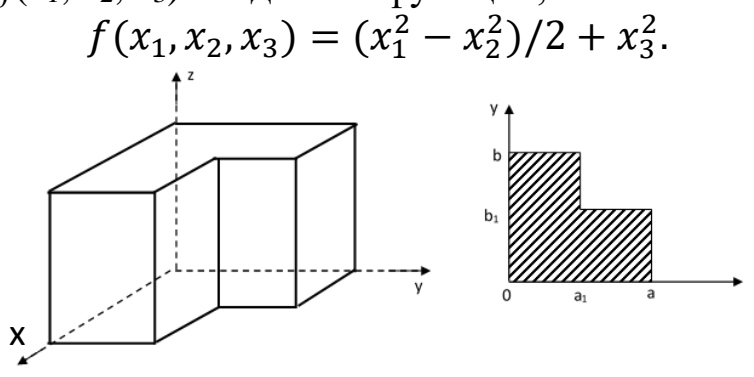

a)

б)

Рис. 1. Геометрическое представление объекта исследования

Сопоставим краевой задаче (21)-(22) вариационную задачу [19] о нахождении минимума функционала

$$
I=\int_{\Omega}(\nabla T)^{2} d \Omega .
$$

Приближенное решение задачи (23) представляется соотношением в виде структурной формулы (GSS)

$$
T(x)=T_{0}+\sum_{i, j}^{n} C_{i j} \chi_{i j}(x),
$$

где $\chi_{i j}(x)=\omega(x) \phi_{i j}(x)-$ система координатных функций,

$\omega(x)=\left[\left(a_{1}^{2}-x_{1}^{2}\right) / 2 a_{1}\right] \&\left[\left(a_{2}^{2}-x_{2}^{2}\right) /\right.$ $\left.2 a_{2}\right] \&\left[\left(a_{3}^{2}-x_{3}^{2}\right) / 2 a_{3}\right]$ - уравнение граничной поверхности $S$ области $\Omega$, символ $\&$ - R-конъюнкция [15-16], $\phi_{i j}(x)$ - полиномы (классические или с локальными носителями), $C_{i j}$ - неопределенные коэффициенты, находящиеся из условий минимума функционала (23),

$$
T_{0}(x)=\left(f_{1} \omega_{2} \omega_{3}+f_{2} \omega_{1} \omega_{3}+\right.
$$




$$
\begin{gathered}
\left.+f_{3} \omega_{2} \omega_{1}\right) /\left(\omega_{2} \omega_{3}+\omega_{2} \omega_{3}+\omega_{2} \omega_{3}\right), \\
f_{1}=\frac{a_{1}^{2}+x_{2}^{2}}{2}-x_{2}^{2}, \\
f_{2}=\frac{a_{2}^{2}+x_{2}^{2}}{2}-x_{3}^{2}, \quad f_{3}=\frac{x_{1}^{2}+x_{2}^{2}}{2}-1, \\
\omega_{1}=\left(a_{1}^{2}-x_{1}^{2}\right), \\
\omega_{2}=\left(a_{2}^{2}-x_{2}^{2}\right), \quad \omega_{3}=\left(a_{3}^{2}-x_{3}^{2}\right) . \\
\text { Результаты решения задачи }(21)-(23)
\end{gathered}
$$
приведены в табл. 1. В верхней строке точное решение [9]

$$
T_{\text {точн }}=\frac{x_{1}^{2}+x_{2}^{2}}{2}-x_{3}^{2},
$$

во второй и третьей строках - значения температур в плоскости $x_{3}=0$, при $n=4$ и $n=10$ соответственно.

Таблица 1 - Значение функиии $T\left(x_{1}, x_{2}, x_{3}\right)$ в некоторых точках области $\Omega$ для плоскости $x_{3}=0$

\begin{tabular}{|l|l|l|l|l|}
\hline \multicolumn{5}{|l|}{$x_{2}$} \\
\hline$x_{1}$ & -0.9 & -0.4 & 0.1 & 0.6 \\
\hline \multirow{4}{*}{-9.9} & 0.810000 & 0.485000 & 0.410000 & 0.585000 \\
& 0.795500 & 0.472322 & 0.399194 & 0.570109 \\
& 0.779681 & 0.479102 & 0.408816 & 0.575791 \\
\hline \multirow{4}{*}{0.4} & 0.485000 & 0.160000 & 0.085000 & 0.260000 \\
& 0.472322 & 0.149948 & 0.079373 & 0.245287 \\
& 0.479102 & 0.154903 & 0.086178 & 0.255433 \\
\hline \multirow{4}{*}{0.1} & 0.410000 & 0.085000 & 0.010000 & 0.185000 \\
& 0.399194 & 0.079373 & 0.009591 & 0.174219 \\
& 0.408816 & 0.086178 & 0.009739 & 0.184921 \\
\hline \multirow{4}{*}{0.6} & 0.585000 & 0.260000 & 0.185000 & 0.360000 \\
& 0.570109 & 0.245287 & 0.174219 & 0.321291 \\
& 0.575791 & 0.255433 & 0.184921 & 0.359315 \\
\hline
\end{tabular}

Большое количество задач теплопроводности для более сложных ограниченных пространственных областей со сложными граничными воздействиями приведены в работах [15-17,20].

Выводы. Разработан подход к решению пространственных задач теплопроводности в областях сложной геометрической формы, основанный на совместном применении теории R-функций и дифференциально-разностного метода.

Дифференциально-разностный метод в сочетании с теорией R-функций расширяет сферу исследования пространственных задач эллиптического типа для неклассических тел, если их граничные поверхности не параллельны координатным плоскостям.

Решения получены в виде ряда, который представляется функциональными соотношениями, состоящими из элементарных функций или суперпозиции элементарных и специальных функций. При этом учитываются на аналитическом уровне граничные условия исходной задачи и геометрия области исследования, описанная в виде аналитического представления. Последнее позволяет провести обширный вычислительный эксперимент для любой области, если заменить область исследования иным аналитическим выражением, не изменяя алгоритма

Рассмотренный подход исследования пространственных задач позволили разработать алгоритмы и вычислительные схемы, с помощью которых были созданы компьютерные модели задач для тел произвольной формы без ограничений на типы граничных условий.

Приведены численные результаты, полученные вышеупомянутым подходом. Достоверность численных результатов проверялась исследованиями сходимости решений.

Данный подход может быть использован как первый этап для изучения термонапряженного состояния конструктивных элементов неклассической геометрической формы [21].

\section{ЛИТЕРАТУРА:}

1. Панферов В.И., Нагорная А.Н., Пашнина Е.Ю. Моделирование и управление тепловым режимом здания. Материалы Международной науч.-техн. конф. «Теоретические основы теплогазоснабжения и вентиляцииџ. М.: Изд-во МГСУ, 2005. C. 143-147.

2. Панферов С.В., Панферов В.И. К задаче параметрической идентификации математической модели теплового режима зданий. Вестник ЮУрГУ. Серия «Строительство и архитектура». 2012. Вып. 14, № 17 (276). C. 73-76.

3. Сидоров В.Н., Ахметов В.К. Математическое моделирование. М.: Изд-во АВС, 2015. $336 \mathrm{c}$.

4. Мазо А.Б. Основы теории и методы расчета теплопередачи: учебное. Казань: Казан. ун-т, 2013. 144 с.

5. Воскобойников Ю. Е. [и др.] Решение инженерных задач в пакете MathCAD: учеб. пособие / под ред. Ю. Е. Воскобойникова. Новосибирск: НГАСУ (Сибстрин), 2013. $120 \mathrm{c}$. 
6. Мацкевич С.М. Численно-аналитическое решение нестационарной задачи теплопроводности в строительных конструкциях: дисс. ... кандидата технических наук: 05.13.18.]. М.: ФГБОУ ВО «Национальный исследовательский Московский государственный строительный университет». 2015. 190 c.

7. Лыков А.В. Теория теплопроводности. М.: Наука, 1986. 696 с.

8. Карслоу Е., Егер Д. Теория теплопроводности. М.: Наука, 1979. 448 с.

9. Тихонов А.Н., Самарский А.А. Уравнения математической физики. М.: Изд. МГУ; Изд. «Наука», 2004. 798 с.

10. Бухтияров В.В. Тепломассообмен. Иваново: Изд-во госуниверситета, 2014. 360 c.

11. Диткин В.А., Прудников А.П. Интегральные преобразования и операционное исчисление. М.: Наука, 1974. 543 с.

12. Самарский А.А., Вабищевич П.Н. Вычислительная теплопередача. М: Едиториал УРСС, 2003. $784 \mathrm{c}$.

13. Зенкевич О. Метод конечных элементов в технике. М.: Мир, 1975. 349 с.

14. Метод конечных элементов в механике твердых тел / Под ред. А.С. Сахарова и И. Альтенбаха. Киев: Вища школа; Лейпциг: Феб Фахбухферлаг, 1982. 420 с.

15. Рвачев В.Л. Теория R-функций и некоторые ее приложения. Киев: Наук. думка, 1982.552 c.

16. Рвачев В.Л., Кравченко В.Ф. Алгебра логики, атомарные функции и вейвлеты. М.: Физмат лит., - 2006.- 416 с.

17. Рвачев В.Л., Слесаренко А.П. Алгебрологические и проекционные методы в задачах теплообмена. Киев: Наук. думка, 1978. $140 \mathrm{c}$.

18. Михлин С.Г. Вариационные методы в математической физике. М.: Наука, 1970. $511 \mathrm{c}$.
19. Михлин С.Г. Численная реализация вариационных методов. М.: Наука, 1966. 348 с.

20. Кравченко В.Ф., Сизова Н.Д. Исследование некоторых задач теплопроводности методом R-функций. Зарубежная радиоэлектроника. 2001. №8. C.41-68.

21. Сізова Н.Д., Гречко Н.В. Визначення термопружного стану стержневої конструкції. Науковий вісник будівництва. 2015. Вип. 81. С.265-270.

СИзова Н.Д. ЧИСЕЛЬНО-АНАЛІТИЧНІ РІШЕННЯ ТЕМПЕРАТУРНИХ ЗАДАЧ В ОБЛАСТЯХ СКЛАДНОЇ ФОРМИ. Наводяться дослідження температурних задач в областях складної геометричної форми, які засновані на спільному застосуванні дифференціально-різницевого підходу й методу R-Функцій (RFM). Рішення температурних задач представлені в аналітичному виді з можливістю визначення чисельних рішень із залученням сучасних методів.

Ключові слова: температурні задачі, моделювання, метод R-Функцій, різницеві схеми, алгоритм, обчислювальний експеримент.

\begin{tabular}{llll} 
Sizova N.D. NUMERICAL-ANALYTICAL \\
SOLUTIONS & \multicolumn{2}{c}{ OF } \\
PROBLEMS IN THEMPRATURE \\
CHE AREAS OF
\end{tabular} COMPLEX FORM. Investigations of temperature problems in the fields of difficult geometric form, which are based on common usage of differential approach and R-function method (RFM) are given. Solutions of temperature problems are presented in analytical form with possibility of determination of numerical solutions with usage of modern methods.

Keyword: temperature problems, modelling, Rfunction method, difference schemes, algorithm, computational experiment. 